\title{
ELEMENTARY EXACT EVALUATION \\ OF INFINITE INTEGRALS OF THE PRODUCT OF SEVERAL SPHERICAL BESSEL FUNCTIONS, POWER AND EXPONENTIAL
}

\author{
BY \\ V. I. FABRIKANT \\ Prisoner \#167932D, Archambault Jail, 242 Montee Gagnon, Ste-Anne-Des-Plaines, Quebec, Canada \\ JON $1 \mathrm{HO}$
}

\begin{abstract}
An elementary analytical method is presented for computation of integrals from zero to infinity involving the product of 3 or more spherical Bessel functions multiplied by an exponential and an arbitrary power. The method is based on the fact that spherical Bessel functions are essentially combinations of elementary functions and that any can be obtained from the function of zero order by an appropriate differentiation.
\end{abstract}

1. Introduction. There are numerous articles devoted to analytical and numerical evaluation of infinite integrals, containing the maximum of three spherical Bessel functions. Such integrals are encountered in nuclear physics (Davies, 1988; Elbaz et al., 1974), in image processing (Baddour, 2010), in antenna design (Qia and others, 2007), particle physics (Anni, et al., 1974), astrophysics and in other fields of science and engineering (Jackson and Maximon, 1972; Chen and Su, 2004).

The results published so far look too cumbersome, the approach is overcomplicated, the final results are expressed in terms of quantum mechanics $3 j$ and $6 j$ coefficients and Legendre functions (Mehrem et al., 1991, 2010). Despite such overcomplication, applicability of these results is quite limited: the sum of subindexes of the spherical Bessel functions should be even and the parameters should be capable of forming a triangle, and even the numbers, expressing order of spherical Bessel functions, should form a triangle. All these limitations are unnecessary.

Yet another overcomplicated approach is presented in (Gebremariam et al., 2010), where the authors compute the infinite integral involving 2 spherical Bessel functions, exponential and power function. They use Mathematica software to first compute an integral from 0 to $y$ and then compute the limit of $y$ tending to infinity.

All this looks very strange, taking into consideration that spherical Bessel functions comprise only elementary functions of sine, cosine and integer powers, and nothing else.

Received November 16, 2011.

2000 Mathematics Subject Classification. Primary 33C55, 81V35.

E-mail address: valery_fabrikant@hotmail.com

(C) 2012 Brown University Reverts to public domain 28 years from publication 
This means that there should be elementary ways to compute integrals of these functions. This elementary approach is presented here.

2. Description of the method. As we know from (Gradshteyn and Ryzhik, 1994) the spherical Bessel function of order $n$ is related to a regular Bessel function as

$$
j_{n}(z)=\frac{\sqrt{\pi}}{\sqrt{2 z}} J_{n+1 / 2}(z) .
$$

Denoting the spherical Bessel function of order zero as

$$
j_{0}(z)=\frac{\sqrt{\pi}}{\sqrt{2 z}} J_{1 / 2}(z)=\frac{\sin z}{z},
$$

we get the following relationship:

$$
j_{n}(z)=(-1)^{n} z^{n} \frac{d^{n} j_{0}(z)}{(z d z)^{n}} .
$$

Taking (2) into consideration, we can rewrite (3) as

$$
j_{n}(z)=(-1)^{n+1} z^{n+1} \frac{d^{n+1} \cos (z)}{(z d z)^{n+1}} .
$$

Suppose we need to compute

$$
I(q, m, n, k ; a, b, c)=\int_{0}^{\infty} t^{q} j_{m}(a t) j_{n}(b t) j_{k}(c t) d t .
$$

Here $m, n$, and $k$ are integers and $q, a, b$, and $c$ are real numbers.

By using (3) we can obtain the result

$$
\begin{aligned}
& I(q, m, n, k ; a, b, c) \\
& =(-1)^{m+n+k} a^{m} b^{n} c^{k} \frac{\partial^{m}}{(a \partial a)^{m}} \frac{\partial^{n}}{(b \partial b)^{n}} \frac{\partial^{k}}{(c \partial c)^{k}} \int_{0}^{\infty} \frac{\sin (a t) \sin (b t) \sin (c t) d t}{a b c t^{m+n+k+3-q}} .
\end{aligned}
$$

We realize that in the general case the integral in (6) is divergent, but we can either regularize it and use the finite part or just compute it formally, having in mind that the differentiation to follow will lead to the correct result. By using the trigonometric identity

$$
\begin{aligned}
\sin (a x) \sin (b x) \sin (c x)= & \frac{1}{4}(-\sin [(a+b+c) x] \\
& +\sin [(a-b+c) x]+\sin [(b+c-a) x]+\sin [(a+b-c) x]),
\end{aligned}
$$

we can reduce the computation of (6) to a simple integral (Gradshteyn and Ryzhik, 1994, formula 3.761.4)

$$
\int_{0}^{\infty} \frac{\sin (s t) d t}{t^{n}}=\cos \left(\frac{\pi n}{2}\right) \Gamma(1-n)|s|^{n-1} \operatorname{sign}(s) .
$$


The substitution of (7) into (6) and the utilization of (8) yields

$$
\begin{aligned}
& I(q, m, n, k ; a, b, c) \\
& =(-1)^{m+n+k} a^{m} b^{n} c^{k} \cos \left(\frac{\pi(m+n+k+3-q)}{2}\right) \frac{\partial^{m}}{(a \partial a)^{m}} \frac{\partial^{n}}{(b \partial b)^{n}} \frac{\partial^{k}}{(c \partial c)^{k}} \\
& \quad \times \frac{\Gamma(q-m-n-k-2)}{4 a b c}\left[|c+a-b|^{m+n+k+2-q} \operatorname{sign}(c+a-b)\right. \\
& \quad+|c-a+b|^{m+n+k+2-q} \operatorname{sign}(c-a+b) \\
& \left.\quad+|a+b-c|^{m+n+k+2-q} \operatorname{sign}(a+b-c)-(c+a+b)^{m+n+k+2-q}\right] .
\end{aligned}
$$

It is presumed in (9), without loss of generality, that $a, b$, and $c$ are positive. The differentiation can be performed either directly or by using the following general formula:

$$
\begin{aligned}
z^{n+1}\left(\frac{\partial}{z \partial z}\right)^{n+1} f(z)= & \sum_{k=0}^{\left[\frac{n}{2}\right]} \frac{\Gamma(n+2 k+1) f^{(n-2 k+1)}(z)}{\Gamma(2 k+1) \Gamma(n-2 k+1)(2 z)^{2 k}} \\
& -\sum_{k=0}^{\left[\frac{n-1}{2}\right]} \frac{\Gamma(n+2 k+2) f^{(n-2 k)}(z)}{\Gamma(2 k+2) \Gamma(n-2 k)(2 z)^{2 k+1}} .
\end{aligned}
$$

Here $[n / 2]$ denotes the integer part of the ratio; $f^{(m)}(z)$ stands for the $m$-th derivative of $f$ with respect to $z$.

We realize additional difficulty in using (9), when $q-m-n-k-2$ is zero or a negative integer, as well as in the case of $m+n+k+3-q$ being an odd integer. We shall need to compute certain limits. The basic limits are related to the following integrals:

$$
\int_{0}^{\infty} \frac{\sin (a t) \sin (b t) \sin (c t) d t}{t}= \begin{cases}\frac{\pi}{4} & \text { when } a-b<c<a+b \\ \frac{\pi}{8} & \text { when } c=a+b \text { or } c=a-b \\ 0 & \text { when } c>a+b \text { or } c<a-b\end{cases}
$$

It is presumed, without loss of generality, that $a>b$. Then

$$
\begin{gathered}
\int_{0}^{\infty} \frac{\sin (a t) \sin (b t) \sin (c t) d t}{t^{2}}=\frac{1}{4}[(c+a+b) \ln (c+a+b)-(c+a-b) \ln |c+a-b| \\
-(c-a+b) \ln |c-a+b|-(a+b-c) \ln |a+b-c|] \\
\int_{0}^{\infty} \frac{\sin (a t) \sin (b t) \sin (c t) d t}{t^{3}}=\frac{\pi}{8}\left[\left(2 a c+2 a b+2 b c-a^{2}-b^{2}-c^{2}\right)\right],
\end{gathered}
$$

when $|a-b|<c<a+b$, and

$$
\begin{gathered}
\int_{0}^{\infty} \frac{\sin (a t) \sin (b t) \sin (c t) d t}{t^{3}}=\frac{\pi a b}{2}, \text { when } c \geq a+b \\
\int_{0}^{\infty} \frac{\sin (a t) \sin (b t) \sin (c t) d t}{t^{3}}=\frac{\pi b c}{2}, \text { when } 0<c \leq|a-b| .
\end{gathered}
$$

The reader should notice that the relevant formula 3.763.4 in Gradshteyn and Ryzhik is in error. 
3. Generalization for the case of four Bessel functions. It is obvious that in the case of 4 spherical Bessel functions formula (6) will take the form

$$
\begin{gathered}
I(q, m, n, k, l ; a, b, c, g) \\
=(-1)^{m+n+k+l} a^{m} b^{n} c^{k} g^{l} \frac{\partial^{m}}{(a \partial a)^{m}} \frac{\partial^{n}}{(b \partial b)^{n}} \frac{\partial^{k}}{(c \partial c)^{k}} \frac{\partial^{l}}{(g \partial g)^{l}} \int_{0}^{\infty} \frac{j_{0}(a t) j_{0}(b t) j_{0}(c t) j_{0}(g t) d t}{t^{m+n+k+l-q}} .
\end{gathered}
$$

The relevant trigonometric relationship to be used here is

$$
\begin{aligned}
& \sin (a x) \sin (b x) \sin (c x) \sin (g x) \\
& =\frac{1}{8}(\cos [(a+b+c+g) x]+\cos [(a+b-c-g) x]+\cos [(a-b+c-g) x] \\
& +\cos [(a-b-c+g) x]-\cos [(-a+b+c+g) x]-\cos [(a-b+c+g) x] \\
& -\cos [(a+b-c+g) x]-\cos [(a+b+c-g) x]),
\end{aligned}
$$

so that the procedure of integration reduces to the following integral (Gradshteyn and Ryzhik, 1994, formula 3.761.9):

$$
\int_{0}^{\infty} \frac{\cos (s t) d t}{t^{n}}=\Gamma(1-n) \sin \left(\frac{\pi n}{2}\right)|s|^{n-1}=\frac{\pi|s|^{n-1}}{2 \Gamma(n) \cos (\pi n / 2)} .
$$

Utilization of (16) and (17) allows us to compute the basic integral

$$
\begin{aligned}
& \int_{0}^{\infty} t^{q} j_{0}(a t) j_{0}(b t) j_{0}(c t) j_{0}(g t) d t=\frac{\pi}{16 \Gamma(4-q) a b c g \cos (\pi q / 2)}\left[|a+b+c+g|^{3-q}\right. \\
& \quad+|a-b-c+g|^{3-q}+|a-b+c-g|^{3-q} \\
& \quad+|a+b-c-g|^{3-q}-|-a+b+c+g|^{3-q}-|a-b+c+g|^{3-q} \\
& \left.\quad-|a+b-c+g|^{3-q}-|a+b+c-g|^{3-q}\right] .
\end{aligned}
$$

In the case of $q$ being an integer, we shall need to compute proper limits. For example, in the case of $q=1$, we get

$$
\begin{aligned}
\int_{0}^{\infty} & t j_{0}(a t) j_{0}(b t) j_{0}(c t) j_{0}(g t) d t \\
= & \frac{1}{16 a b c g}\left[(a+b+c+g)^{2} \ln |a+b+c+g|+(a+b-c-g)^{2} \ln |a+b-c-g|\right. \\
& \quad+(a-b-c+g)^{2} \ln |a-b-c+g|+(a-b+c-g)^{2} \ln |a-b+c-g| \\
& \quad-(-a+b+c+g)^{2} \ln |-a+b+c+g| \\
& -(a-b+c+g)^{2} \ln |a-b+c+g|-(a+b-c+g)^{2} \ln |a+b-c+g| \\
& \left.-(a+b+c-g)^{2} \ln |a+b+c-g|\right] .
\end{aligned}
$$

In the case of $q=3$ the result is

$$
\begin{gathered}
\int_{0}^{\infty} t^{3} j_{0}(a t) j_{0}(b t) j_{0}(c t) j_{0}(g t) d t \\
=\frac{1}{16 a b c g} \ln \frac{|-a+b+c+g \| a-b+c+g||a+b-c+g||a+b+c-g|}{|a+b+c+g||a+b-c-g||a-b+c-g||a-b-c+g|} .
\end{gathered}
$$

The cases of $q=0$ and $q=2$ are trivial. 
4. The case of exponential and 3 spherical Bessel functions. We are now dealing with the integral $I_{e}(p, q, m, n, k ; a, b, c)=\int_{0}^{\infty} \exp (-p t) t^{q} j_{m}(a t) j_{n}(b t) j_{k}(c t) d t$. Using the same logic as before, we come to the result

$$
\begin{aligned}
I_{e}(p, q, m, n, k ; a, b, c)= & (-1)^{m+n+k} a^{m} b^{n} c^{k} \frac{\partial^{m}}{(a \partial a)^{m}} \frac{\partial^{n}}{(b \partial b)^{n}} \frac{\partial^{k}}{(c \partial c)^{k}} \\
& \times \int_{0}^{\infty} \exp (-p t) \frac{j_{0}(a t) j_{0}(b t) j_{0}(c t)}{t^{m+n+k-q}} d t .
\end{aligned}
$$

The basic integral can be found in (Gradshteyn and Ryzhik, 1994, formula 3.944.5)

$$
\int_{0}^{\infty} \exp (-p t) \frac{\sin (s t) d t}{t^{n}}=\frac{\Gamma(1-n)}{\left(p^{2}+s^{2}\right)^{(1-n) / 2}} \sin \left((1-n) \tan ^{-1} \frac{s}{p}\right) .
$$

The application of (23) to (22) gives us

$$
\begin{aligned}
\int_{0}^{\infty} & t^{q} \exp (-p t) j_{0}(a t) j_{0}(b t) j_{0}(c t) d t \\
= & \frac{\pi}{4 a b c \Gamma(3-q) \sin (\pi q)}\left(-\left[p^{2}+(c+b+a)^{2}\right]^{(2-q) / 2} \sin \left\{(q-2) \tan ^{-1}[(c+b+a) / p]\right\}\right. \\
& +\left[p^{2}+(c+b-a)^{2}\right]^{(2-q) / 2} \sin \left\{(q-2) \tan ^{-1}[(c+b-a) / p]\right\} \\
& +\left[p^{2}+(c+a-b)^{2}\right]^{(2-q) / 2} \sin \left\{(q-2) \tan ^{-1}[(c+a-b) / p]\right\} \\
& \left.+\left[p^{2}+(a+b-c)^{2}\right]^{(2-q) / 2} \sin \left\{(q-2) \tan ^{-1}[(a+b-c) / p]\right\}\right) .
\end{aligned}
$$

In the case of $q$ being an integer, proper limits need to be computed. For example, for $q=1$ the result is

$$
\begin{aligned}
\int_{0}^{\infty} & t \exp (-p t) j_{0}(a t) j_{0}(b t) j_{0}(c t) d t \\
= & \frac{1}{8 a b c}\left(( c + b + a ) \operatorname { l n } \left[p^{2}\right.\right. \\
& \left.\quad+(c+b+a)^{2}\right]+2 p \tan ^{-1}[(c+b+a) / p] \\
& \quad-(c+b-a) \ln \left[p^{2}+(c+b-a)^{2}\right]-2 p \tan ^{-1}[(c+b-a) / p] \\
& \quad-(c+a-b) \ln \left[p^{2}+(c+a-b)^{2}\right]-2 p \tan ^{-1}[(c+a-b) / p] \\
& \left.-(a+b-c) \ln \left[p^{2}+(a+b-c)^{2}\right]-2 p \tan ^{-1}[(a+b-c) / p]\right) .
\end{aligned}
$$

In the case of $q=2$, we obtain

$$
\begin{aligned}
& \int_{0}^{\infty} t^{2} \exp (-p t) j_{0}(a t) j_{0}(b t) j_{0}(c t) d t=\frac{1}{4 a b c}\left(-\tan ^{-1}[(c+b+a) / p]\right. \\
& \left.\quad+\tan ^{-1}[(c+b-a) / p]+\tan ^{-1}[(c+a-b) / p]+\tan ^{-1}[(a+b-c) / p]\right) .
\end{aligned}
$$

The case of 4 spherical Bessel functions can be resolved in the same manner, as indicated in (17), and the basic integral here will be

$$
\int_{0}^{\infty} \exp (-p t) \frac{\cos (s t) d t}{t^{n}}=\frac{\Gamma(1-n)}{\left(p^{2}+s^{2}\right)^{(1-n) / 2}} \cos \left((1-n) \tan ^{-1} \frac{s}{p}\right) .
$$


In general, when we have an odd number of spherical Bessel functions, the basic integral is (23); and in the case of an even number of spherical Bessel functions, the basic integral is $(27)$.

5. Examples. We took as examples some of those computed in (Mehrem, 2010), as well as some which cannot be computed by his method:

$$
\begin{aligned}
I(2,1,0,1 ; a ; b ; c) & =\int_{0}^{\infty} t^{2} j_{1}(a t) j_{0}(b t) j_{1}(c t) d t \\
& =(-1)^{2} \frac{\partial}{\partial a} \frac{\partial}{\partial c} \int_{0}^{\infty} j_{0}(a t) j_{0}(b t) j_{0}(c t) d t .
\end{aligned}
$$

Utilization of (13)-(15) in (28) gives us

$$
\begin{aligned}
I(2,1,0,1 ; a ; b ; c) & =\frac{\partial}{\partial a} \frac{\partial}{\partial c}\left[\frac{\pi}{8 a b c}\left(\left(2 a c+2 a b+2 b c-a^{2}-b^{2}-c^{2}\right)\right)\right] \\
& =\frac{\pi}{8 a^{2} b c^{2}}\left(a^{2}-b^{2}+c^{2}\right)
\end{aligned}
$$

for $|a-b|<c<a+b$. The result is one half of that given by (29) at the boundaries $c=|a-b|$ or $c=a+b$, and it is zero otherwise.

In a similar manner, we get

$$
\begin{aligned}
I(2,1,1,0 ; a ; b ; c) & =\int_{0}^{\infty} t^{2} j_{1}(a t) j_{1}(b t) j_{0}(c t) d t \\
& =\frac{\partial}{\partial a} \frac{\partial}{\partial b}\left[\frac{\pi}{8 a b c}\left(\left(2 a c+2 a b+2 b c-a^{2}-b^{2}-c^{2}\right)\right)\right]=\frac{\pi}{8 a^{2} b^{2} c}\left(a^{2}+b^{2}-c^{2}\right),
\end{aligned}
$$

for $|a-b|<c<a+b$. The result is one half of that given by (30) at the boundaries $c=|a-b|$ or $c=a+b$, and it is zero otherwise.

The next example is

$$
\begin{aligned}
& I(1,1,1,1 ; a, b, c)=\int_{0}^{\infty} t j_{1}(a t) j_{1}(b t) j_{1}(c t) d t \\
& =(-1)^{3} \cos \left(\frac{5 \pi}{2}\right) \frac{\partial}{\partial a} \frac{\partial}{\partial b} \frac{\partial}{\partial c} \frac{\Gamma(-4)}{4 a b c}\left[|c+a-b|^{4} \operatorname{sign}(c+a-b)\right. \\
& \left.\quad+|c-a+b|^{4} \operatorname{sign}(c-a+b)+|a+b-c|^{4} \operatorname{sign}(a+b-c)-(c+a+b)^{4}\right] .
\end{aligned}
$$

Simplification of (31) for the case $|a-b|<c<a+b$ yields

$$
I(1,1,1,1 ; a, b, c)=\frac{\pi}{32 a^{2} b^{2} c^{2}}\left(c^{2}-(a-b)^{2}\right)\left((a+b)^{2}-c^{2}\right),
$$

and we get zero otherwise. The next example yields

$$
I(0,0,0,2 ; a, b, c)=\frac{\pi}{32 a b c^{3}}\left(c^{2}-(a-b)^{2}\right)^{2}
$$

for the case $|a-b|<c<a+b$. The result is

$$
I(0,0,0,2 ; a, b, c)=\frac{\pi}{4 c^{3}}\left(c^{2}-a^{2}-b^{2}\right)
$$

for $c \geq a+b$, and we get zero otherwise. We consider

$$
I(0,1,1,2 ; a, b, c)=\int_{0}^{\infty} j_{1}(a t) j_{1}(b t) j_{2}(c t) d t .
$$


We obtain

$$
I(0,1,1,2 ; a, b, c)=\frac{\pi\left[c^{2}-(a-b)^{2}\right]\left[(a+b)^{2}+2 a b-c^{2}\right]}{192 a^{2} b^{2} c^{3}}
$$

for the case $|a-b|<c<a+b$. The result is

$$
I(0,1,1,2 ; a, b, c)=\frac{\pi a b}{6 c^{3}}
$$

for $c \geq a+b$, and we get zero otherwise. A somewhat more complicated example is

$$
I(0,1,0,3 ; a, b, c)=\int_{0}^{\infty} j_{1}(a t) j_{0}(b t) j_{3}(c t) d t .
$$

The result is

$$
I(0,1,0,3 ; a, b, c)=\frac{\pi\left[c^{2}-(a-b)^{2}\right]\left[(a-b)(5 a+b)+c^{2}\right]}{192 a^{2} b c^{4}}
$$

for the case $|a-b|<c<a+b$. The result is

$$
I(0,1,0,3 ; a, b, c)=\frac{\pi a\left[3\left(c^{2}-a^{2}\right)-5 b^{2}\right]}{12 c^{4}}
$$

for $c \geq a+b$, and we get zero otherwise.

The above results are in agreement with (Mehrem et al., 2010), in the interval $|a-b|<$ $c<a+b$, except (33)-(40), where Mehrem's result is in error at the boundary $c=a+b$ (there should be no factor $1 / 2$ there). Mehrem was also unable to give the result at $c>a+b$. Here is an example which cannot be computed by the method described there:

$$
I(0,1,1,1 ; a, b, c)=\int_{0}^{\infty} j_{1}(a t) j_{1}(b t) j_{1}(c t) d t .
$$

Formal use of (6) leads to

$$
\begin{aligned}
& I(0,1,1,1 ; a, b, c) \\
& =-\lim _{q \rightarrow 0}\left[\frac { \partial } { \partial a } \frac { \partial } { \partial b } \frac { \partial } { \partial c } \frac { \pi } { 8 a b c \operatorname { s i n } [ ( 3 - q / 2 ) \pi ] \Gamma ( 6 - q ) } \left((c+a-b)^{5-q} \operatorname{sign}(c+a-b)\right.\right. \\
& \left.\left.-(c+a+b)^{5-q}+|c-a+b|^{5-q} \operatorname{sign}(c-a+b)+|a+b-c|^{5-q} \operatorname{sign}(a+b-c)\right)\right] .
\end{aligned}
$$

The final result is

$$
\begin{aligned}
& I(0,1,1,1 ; a, b, c) \\
& =\frac{1}{120 a^{2} b^{2} c^{2}}\left[( - a + b + c ) ^ { 2 } \left[(-a+b+c)^{3}-5(b+c)\left(a^{2}+b c\right)\right.\right. \\
& \left.\quad+5 a\left(b^{2}+c^{2}\right)\right] \ln |-a+b+c| \\
& \quad+(a-b+c)^{2}\left[(a-b+c)^{3}+5(b-c)\left(a^{2}-b c\right)-5 a\left(b^{2}+c^{2}\right)\right] \ln |a-b+c| \\
& \quad+(a+b-c)^{2}\left[(a+b-c)^{3}-5(b-c)\left(a^{2}-b c\right)-5 a\left(b^{2}+c^{2}\right)\right] \ln |a+b-c| \\
& \quad-(a+b+c)^{2}\left[(a+b+c)^{3}\right. \\
& \left.\left.\quad-5(b+c)\left(a^{2}+b c\right)-5 a\left(b^{2}+c^{2}\right)\right] \ln |a+b+c|-4 a b c\left(a^{2}+b^{2}+c^{2}\right)\right] .
\end{aligned}
$$


Here is an example of integration of 4 spherical Bessel functions:

$$
\begin{aligned}
I(2,0,1,2,3 ; a, b, c, g)=\int_{0}^{\infty} t^{2} j_{0}(a t) j_{1}(b t) j_{2}(c t) j_{3}(g t) d t \\
=\lim _{q \rightarrow 2}\left[c^{2} g^{3} \frac{\partial}{\partial b} \frac{\partial^{2}}{(c \partial c)^{2}} \frac{\partial^{3}}{(g \partial g)^{3}} \int_{0}^{\infty} \frac{j_{0}(a t) j_{0}(b t) j_{0}(c t) j_{0}(g t) d t}{t^{6-q}}\right] \\
=c^{2} g^{3} \frac{\partial}{\partial b} \frac{\partial^{2}}{(c \partial c)^{2}} \frac{\partial^{3}}{(g \partial g)^{3}}\left[\frac { \pi } { 1 6 \cdot 7 ! a b c g } \left(|a+b+c+g|^{7}+|a-b-c+g|^{7}+|a-b+c-g|^{7}\right.\right. \\
\quad+|a+b-c-g|^{7}-|-a+b+c+g|^{7} \\
\left.\left.\quad-|a-b+c+g|^{7}-|a+b-c+g|^{7}-|a+b+c-g|^{7}\right)\right] .
\end{aligned}
$$

The results would be quite different, depending on the relative value of the parameters $a, b, c, g$. For example, when any of the parameters is greater than the sum of the remaining 3 , the result is zero. When parameters $a, b$ and $c$ can form a triangle and $g$ is smaller that any difference between $a, b$ and $c$, the result is

$$
I(2,0,1,2,3 ; a, b, c, g)=\frac{\pi g^{3}}{140 a b^{2} c^{3}} .
$$

In the case of very small $c$ and $a$, with $b$ and $g$ capable of forming a triangle, the result is

$$
I(2,0,1,2,3 ; a, b, c, g)=\frac{\pi c^{2}\left[35\left(b^{2}-a^{2}\right)-5 c^{2}+7 g^{2}\right]}{280 a b^{2} g^{4}} .
$$

In the case of very small $b$ and $a$, with $c$ and $g$ capable of forming a triangle, the result is

$$
\begin{aligned}
& I(2,0,1,2,3 ; a, b, c, g) \\
& \quad=\frac{\pi b\left\{15 b^{4}+14 b^{2}\left(15 a^{2}-5 c^{2}-3 g^{2}\right)+35\left[5 a^{4}-2 a^{2}\left(5 c^{2}+3 g^{2}\right)+5 c^{4}+2 c^{2} g^{2}+g^{4}\right]\right\}}{1120 a c^{3} g^{4}} .
\end{aligned}
$$

The general case is quite unwieldy and is not written explicitly. Now we turn to examples containing exponential function. We found just a couple of examples in the literature containing 2 spherical Bessel functions. One such example is (Gebremariam et al., 2010)

$$
I_{e}(p,-1,1,1 ; 1,1)=\int_{0}^{\infty} \exp (-p t) \frac{j_{1}(t) j_{1}(t)}{t} d t .
$$

For utilization of our method, we need to take a more general integral, namely

$$
I_{e}(p,-1,1,1 ; a, b)=\int_{0}^{\infty} \exp (-p t) \frac{j_{1}(a t) j_{1}(b t)}{t} d t
$$

Using (22), we get

$$
I_{e}(p,-1,1,1 ; a, b)=\frac{\partial}{\partial a} \frac{\partial}{\partial b} \int_{0}^{\infty} \exp (-p t) \frac{j_{0}(a t) j_{0}(b t)}{t^{3}} d t
$$

Utilization of (27) leads to

$$
\begin{aligned}
& I_{e}(p,-1,1,1 ; a, b)=\lim _{n \rightarrow 5}\left[\frac { \partial } { \partial a } \frac { \partial } { \partial b } \left(\frac { \pi } { 4 8 a b \operatorname { s i n } ( \pi n ) } \left\{\left[p^{2}+(a-b)^{2}\right]^{(n-1) / 2}\right.\right.\right. \\
& \left.\left.\times \cos \left[(1-n) \tan ^{-1}((a-b) / p)\right]-\left[p^{2}+(a+b)^{2}\right]^{(n-1) / 2} \cos \left[(1-n) \tan ^{-1}((a-b) / p)\right]\right)\right] .
\end{aligned}
$$


Necessary simplifications in (50) give us

$$
\begin{aligned}
& I_{e}(p,-1,1,1 ; a, b)=\frac{1}{96 a^{2} b^{2}}\left[4 a b\left(3\left(a^{2}+b^{2}\right)-p^{2}\right)+16\left(a^{3}-b^{3}\right) p \tan ^{-1}\left(\frac{a-b}{p}\right)\right. \\
& \left.-16\left(a^{3}+b^{3}\right) p \tan ^{-1}\left(\frac{a+b}{p}\right)+\left(3\left(a^{2}-b^{2}\right)^{2}-6\left(a^{2}+b^{2}\right) p^{2}-p^{4}\right) \ln \left(\frac{(a-b)^{2}+p^{2}}{(a+b)^{2}+p^{2}}\right)\right] .
\end{aligned}
$$

In the case of $a=b=1$, formula (51) is in agreement with the result of (Gebremariam et al, 2010). Additional examples of computations of integrals of 3 spherical Bessel functions and an exponential are given above in (24)-(26).

Conclusion. A general and elementary method has been developed, which for the first time allows us to compute infinite integrals consisting of an arbitrary number of spherical Bessel functions multiplied by arbitrary power and an exponential. In each case the result is expressible in terms of elementary functions. Unlike previous work in this field, no limitations are imposed either on the order of spherical Bessel functions or on the relations between factors in their arguments.

Acknowledgement. The author is grateful to his son Isaac for the performance of various computations and the work of preparation of the manuscript for publication.

\section{References}

[1] R. Anni and L. Taffara, DWBA analysis of heavy ion transfer reactions, Nuovo Cimento, 1974, Vol. A22, pp. 11-24.

[2] N. Baddour, Operational and convolution properties of three-dimensional Fourier transforms in spherical polar coordinates. Journal of Optical Society of America, Series A, 2010, Vol. 27, pp. 21442155.

[3] J. Chen and J. Su, Glueball spectrum based on rigorous three-dimensional relativistic equation for two-gluon bound states II: calculation of glueball spectrum, Physics Reviews, 2004, Vol. D69, 076003.

[4] K.T.R Davies, Complex-plane method for evaluating highly oscillatory integrals in nuclear physics, J. Phys. G: Nucl. Phys., 1988, Vol. 14, pp. 973-994.

[5] E. Elbaz, J. Meyer, and R. Nahabetian, On the expansion of a function sum of two vectors as appearing in the recoil effect in nuclear transfer reaction, Lett. Nuovo Cimento, 1974, Vol. 10, pp. $417-421$.

[6] B. Gebremariam, T. Duquet and S.K. Bogner, Symbolic integration of a product of two spherical Bessel functions with an additional exponential and polynomial factor, Computer Physics Communications, 2010, Vol. 181, pp. 1136. MR2644952

[7] I.S. Gradshteyn and I.M. Ryzhik, Tables of Integrals, Series and Products. Academic Press Inc., New York, 1994. MR1243179

[8] A.D. Jackson and L. Maximon, Integrals of products of Bessel functions, SIAM J. Math. Anal., 1972, Vol. 3, pp. 446-460. MR0311958 (47:520)

[9] R. Mehrem, J.T. Londergan and M.H. Macfarlane, Analytic expressions for integrals of products of spherical Bessel functions, J. Phys. A: Math. Theor., 1991, Vol. 24, pp. 1435-1453. MR:1121820 (92h:33011)

[10] R. Mehrem and A. Hohenegger, A generalization for the infinite integrals over three spherical Bessel functions, J. Phys. A: Math. Theor., 2010, Vol. 43, p. 455204. MR2733847 (2011k:33012)

[11] Cheng-Wei Qiu, Le-Wei Li, Saïd Zouhdi, Tat-Soon Teo, and Qun Wu, On the integral identities consisting of two spherical Bessel Functions. IEEE Transactions on Antennas and Propagation, Vol. 55, No. 1, pp. 240-244. MR2289746 (2008b:33012) 